\title{
Effect of V-Go Versus Multiple Daily Injections on Glycemic Control, Insulin Use, and Diabetes Medication Costs Among Individuals with Type 2 Diabetes Mellitus
}

\author{
Amit D. Raval, PhD; Matthew H. Nguyen, PharmD; Siting Zhou, PhD; \\ Michael Grabner, PhD; John Barron, PharmD; and Ralph Quimbo, MA
}

\begin{abstract}
BACKGROUND: Coping with discomfort and the uncertainties of daily adjustments are prominent challenges confronting individuals with type 2 diabetes mellitus (T2DM) who require multiple daily injections (MDI) of insulin. For this growing population, wearable, disposable devices capable of delivering consistent and sustained doses of basal-bolus therapy may help to alleviate concerns and improve outcomes. However, studies on the comparative effectiveness of new, innovative delivery systems versus MDI on insulin requirements, glycemic control, and health care costs are sparse.
\end{abstract}

OBJECTIVE: To examine glycemic control, insulin use, and diabetes medication costs for users of the V-Go Wearable Insulin Delivery device compared with MDI insulin therapy among individuals with T2DM in a commercially insured population in the United States.

METHODS: This retrospective cohort study queried administrative claims data from the HealthCore Integrated Research Database from July 1, 2011, through July 31, 2017. Cohorts included individuals with T2DM aged 21-80 years either newly initiating V-Go or using MDI for basal/bolus insulin. The date of earliest claim for V-Go prescription fill or for bolus insulin was defined as the index date, depending on the cohort. Previous insulin therapy was required in both cohorts. Baseline hemoglobin A1c (A1c) values were identified during the 6 months before and 15 days after the index date; results closest to 12 months after the index date were selected as follow-up. Insulin use and diabetes medication cost data were examined during the 6 months baseline and the second half of the 1-year follow-up. V-Go and MDI users were 1:1 matched on baseline insulin exposure, A1c level, and other characteristics of interest. Univariate and multivariate tests were used to compare follow-up outcomes.

RESULTS: Matched cohorts included 118 well-balanced pairs (mean age: 56 years; mean baseline A1c: $9.2 \%$ ). During follow-up, both cohorts experienced improvements in glycemic control relative to baseline $(\%$ with A1c $\leq 9 \%$, baseline/follow-up: V-Go 49/69, $P<0.001$; MDI 50/60, $P=0.046$ ). With similar baseline insulin prescription fills and diabetes medication costs, V-Go users required fewer insulin prescription fills (mean change: -0.8 vs. +1.8 fills, $P<0.001 ;-17 \%$ vs. $+38 \%$ ); had a smaller increase in diabetes medication costs (mean change in 2016 USD: \$341 vs. $\$ 1,628, P=0.012 ;+10 \%$ vs. $+47 \%$ ); and a decrease in insulin total daily dose (mean change in insulin units per day: -29.2 vs. $+5.8, P<0.001 ;-21 \%$ vs. $+4 \%$ ), compared with MDI users, during the last 6 months of follow-up. CONCLUSIONS: This study was the first to evaluate clinical and economic outcomes associated with the use of V-Go for up to a 1-year follow-up period. Relative to MDI users, V-Go users had similar glycemic control but lower insulin use and lower diabetes medication costs during follow-up.
V-Go therapy may provide an opportunity to improve quality measures more cost-effectively in people with T2DM who require basal-bolus therapy.

J Manag Care Spec Pharm. 2019;25(10):1111-23

Copyright $\odot 2019$, Academy of Managed Care Pharmacy. All rights reserved.

\section{What is already known about this subject}

Despite solid guideline recommendations and use of long-acting basal insulin to achieve and maintain glycemic control, 24\%-77\% of insulin users fail to attain adequate glycemic control in the long term. - A common insulin regimen entails multiple daily injections (MDI) of basal \pm bolus products, but the effectiveness of this approach may be stymied by poor adherence attributable to concerns about insulin treatment itself, as well as fear of needles and other factors associated with delivery by injection.

A handful of small, short-term studies have reported that individuals with diabetes have experienced better glycemic control and incurred lower costs with the use of V-Go, a novel insulin delivery device.

\section{What this study adds}

This study examined matched cohorts of individuals who received insulin treatment with the V-Go insulin delivery device or MDI and evaluated glycemic control, insulin use, and diabetes medication costs for up to a year of follow-up.

While both cohorts experienced improvements in glycemic control, V-Go users had significantly fewer prescriptions fills for insulin, a smaller increase in diabetes-related medication costs, and a decrease in their total daily dose of insulin.

This study demonstrated the effectiveness of V-Go in a real-world population for an extended duration relative to patients receiving MDI, the standard of care.

T he American Diabetes Association (ADA) guidelines recommend the use of insulin by individuals who experience inadequate type 2 diabetes mellitus (T2DM) control with oral diabetes medications. ${ }^{1}$ Subcutaneous longacting basal insulin preparations are usually administered to sustain insulin levels over the entire day for improved glycemic control. While the initiation of basal insulin often provides 
tighter glycemic control, previous studies have shown that 24\%$77 \%$ of individuals fail to achieve adequate long-term glycemic control with basal insulin therapy alone. ${ }^{1-5}$ For ongoing management, ADA guidelines recommend different options, including the addition of either multiple daily injections (MDI) of basal-bolus insulin or premixed insulin therapy. Basal-bolus insulin therapies are preferred as they more closely simulate physiological insulin secretion. ${ }^{1}$

MDI of insulin via vial/syringe or pen can cause considerable discomfort and typically requires substantial individual commitment to self-management and daily living adjustments. ${ }^{1}$ For these and other reasons, adherence to MDI therapy remains poor. ${ }^{6-8}$ Suboptimal adherence to MDI may lead to an increase in insulin resistance, eventually requiring larger insulin doses to address glycemic goals. ${ }^{9-11}$ Left untreated, individuals with diabetes may develop complications, experience poor clinical outcomes, and incur greater health care costs. ${ }^{12,13}$ Effective interventions, including innovative medication delivery devices, improve the management of individuals with T2DM treated with insulin. ${ }^{14,15}$

The V-Go Wearable Insulin Delivery device (V-Go, Valeritas) has a novel mechanism for the administration of basal-bolus therapy. This mechanical device has no electronics, is worn like a patch, and delivers continuous subcutaneous fast-acting U-100 insulin (lispro or aspart) in preset basal rates during a 24-hour period, plus on-demand bolus dosing, circumventing the need for MDI. The device is manually filled with insulin and then attached to a selected area on the patient's skin. To initiate insulin flow, the patient pushes a button linked to a delivery needle. A filled V-Go device is worn by the patient for 24 hours, after which the needle is retracted to remove the disposable device from the body. The process is repeated every 24 hours. ${ }^{16}$

A 4-month pragmatic trial that compared V-Go with standard treatment optimization showed that V-Go users attained better glycemic control and required less insulin. ${ }^{17}$ Using electronic medical record (EMR) data, Sutton et al. (2018) reported that V-Go users $(\mathrm{N}=103)$ had significant reductions in glycemic levels at all follow-ups and, at the end of the 14-month study, significant reductions in insulin use, measured as total daily dose (TDD), and lower per-patient per-month costs; however, the study did not include a comparator cohort. ${ }^{18}$ Also using EMR data, Everitt et al. (2018) compared 108 patients (85 using V-Go; 23 using conventional insulin delivery) who were persistent to therapy for $\geq 5$ months; V-Go patients experienced significant improvements in hemoglobin Alc (Alc) and TDD relative to the comparators over the approximately 8 months of follow-up. ${ }^{19}$ While building on this evidence, additional studies using different data sources, longer time horizons, and comparator groups are needed for a better understanding of the real-world effectiveness of V-Go relative to MDI. ${ }^{20-24}$
The primary objective of this study was to evaluate changes in Alc levels and the achievement of glycemic control among V-Go and MDI users up to a period of 1 year in a commercially insured, real-world population. The secondary objective was to compare the effect of V-Go versus MDI on insulin use patterns, estimated insulin TDD, and diabetes-related medication costs.

\section{Methods}

\section{Study Design and Data Source}

This retrospective, observational cohort study compared outcomes for V-Go versus MDI treatment among individuals with T2DM who had previous insulin therapy. Pharmacy and medical claims, as well as laboratory result data, were taken from the HealthCore Integrated Research Database (HIRD) for the study period July 1, 2011-July 31, 2017. The HIRD contains administrative claims data from enrollees in 14 commercial health plans across the United States. The claims data in the HIRD are integrated across a broad spectrum of sources and types, including professional claims, facility claims, outpatient pharmacy claims, outpatient laboratory results, and enrollment information, among others. The laboratory results included electronic data for Alc tests supplied by several participating outpatient laboratories and were available for a subset of patients. Researchers only accessed data in the format of a limited dataset for which a data use agreement was in place with the covered entities, in compliance with the Health Insurance Portability and Accountability Act (HIPAA) Privacy Rule.

\section{Study Population}

V-Go Cohort. Individuals aged 21-80 years with $\geq 1$ pharmacy fill for V-Go during January 1, 2012-April 30, 2017 (intake period) were selected. The use of this device was identified from National Drug Code numbers available in the pharmacy claims (Appendix A, available in online article). The date of the earliest pharmacy fill of V-Go was defined as the index date and represented the first use of V-Go to modify insulin therapy. Medical conditions (e.g., comorbidities) were confirmed by the presence of $\geq 1$ medical claim with International Classification of Diseases, Ninth/Tenth Revision, Clinical Modification codes. For inclusion, patients were required to have $\geq 6$ months pre-index (baseline) continuous medical and pharmacy plan enrollment to ensure previous insulin exposure and to identify their clinical characteristics. Only individuals with $\geq 1$ claim for T2DM and $\geq 1$ claim for any insulin (except U-500 insulin or bolus insulin alone) during the 6-month baseline period were included; individuals using any insulin pump system during the study period were excluded (codes used to identify insulin fills and pump use as well as other medications are listed in Appendix A). Patients were excluded if they were pregnant, had secondary diabetes, or end-stage renal disease during the 6-month baseline period. No minimum health plan enrollment after the index date was required at this stage. 
Effect of V-Go Versus Multiple Daily Injections on Glycemic Control, Insulin Use, and Diabetes Medication Costs Among Individuals with Type 2 Diabetes Mellitus

\section{FIGURE 1 Study Design to Balance Previous Insulin Exposure Among V-Go and MDI Users}

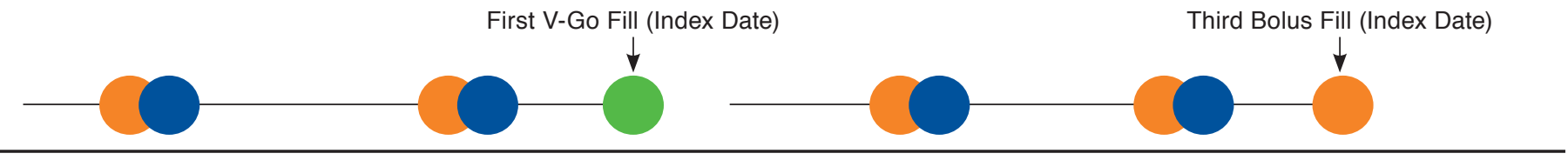

Subset: Previous Basal Insulin Regimen

Matched to

Subset: Previous Basal Insulin Regimen

First V-Go Fill (Index Date)
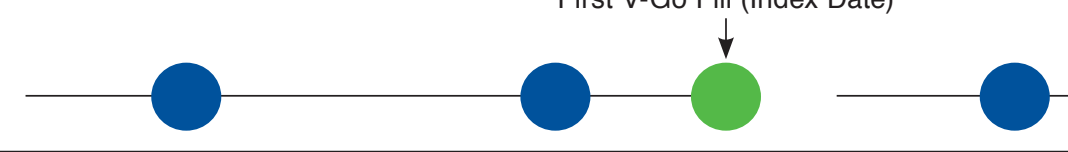

First Bolus Fill (Index Date)

Subset: Previous Premixed Insulin

Matched to

Subset: Previous Premixed Insulin

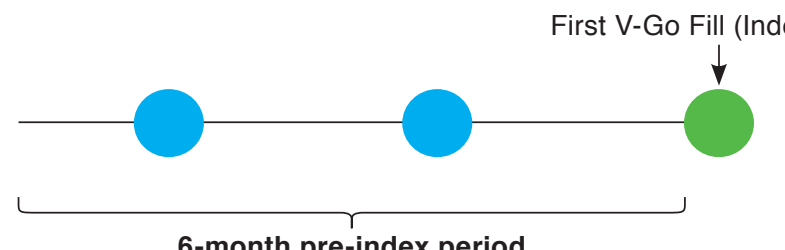

6-month pre-index period

Date)

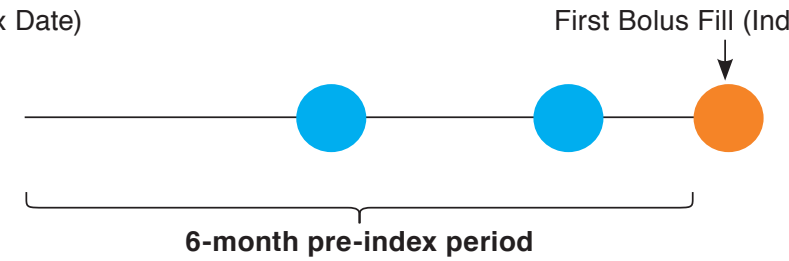

First Bolus Fill (Index Date)

\section{V-Go Basal Bolus Premixed}

Note: This figure explains how MDI patients were matched to V-Go patients based on similar previous insulin regimens. We used 3 scenarios: previous basal-bolus insulin regimens, previous basal insulin-only regimens, and previous premixed insulin regimens. For example, in the previous basal insulin-only scenario, MDI patients with $\geq 2$ pharmacy fills for basal insulin during 6 months before the index date (date of first fill of bolus insulin) were identified and matched to V-Go patients who also had $\geq 2$ pharmacy fills of basal insulin during 6 months before the index date, using propensity score matching. MDI = multiple daily injections.

MDI (Basal-Bolus) Cohort. V-Go users usually follow a basalbolus regimen, and the comparator MDI group was selected to exhibit equivalent insulin regimen patterns (Figure 1) based on previous insulin exposure information and literature-based insulin usage patterns among V-Go users. ${ }^{17,18,20-24}$ Inclusion in the MDI (basal-bolus) cohort required $\geq 2$ pharmacy fills of basal insulin and $\geq 2$ pharmacy fills of bolus insulin within 6 months of each other anytime during the intake period and no V-Go fill during the study period. The index date was defined as the date of the earliest claim for bolus insulin. As in the V-Go cohort, for inclusion, patients were required to have $\geq 6$ months of pre-index (baseline) plan enrollment and $\geq 1$ claim for T2DM at baseline. They also needed to have $\geq 1$ claim for basal-bolus, basal only, or premixed insulin during the 6 months baseline period (3 distinct subgroups were identified within the MDI cohort based on these observed insulin fills, see Figure 1). Patients were excluded if they had any claims for U-500 insulin or insulin pumps at any time during the study period, or for pregnancy, secondary diabetes, or end-stage renal disease at baseline. No minimum health plan enrollment after the index date was required at this stage.

\section{Outcome Measures}

Glycemic Control. Glycemic control was analyzed in subjects who had available Alc data that included $\geq 1$ laboratory result during the baseline period ( 6 months before and 15 days after the index date) and $\geq 1$ result within 1 year of follow-up (3 to 13 months after the index date). To determine glycemic control, the mean change in Alc from baseline to follow-up was compared, and the percentage of patients with Alc $\leq 9 \%$ was evaluated. This cutoff aligns with the National Committee for Quality Assurance Health Effectiveness Data and Information Set measure, which defines poor control as Alc $>9 \% .{ }^{25}$ 
Insulin Use and Diabetes-Related Medication Costs. For the analysis of insulin use and diabetes-related medication costs, patients were required to have $\geq 12$ months of postindex enrollment (follow-up year) with continuous medical and pharmacy coverage, along with the other inclusion criteria. The evaluation of insulin use and diabetes-related medication costs was based on the 6-month baseline period and the last 6 months (Months 7-12) of the follow-up year. Follow-up outcomes were assessed only from Months 7-12 to allow for a treatment adjustment period and to align the duration of observation for the baseline and follow-up periods.

Insulin use was measured via (a) the number of pharmacy claims (fills) for any insulin and (b) the estimated insulin TDD. Insulin TDD, in units per day, was calculated from the number of insulin units dispensed divided by the days of insulin supply, as listed on the last fills closest to the index date during baseline and closest to the end of follow-up. The use of fillbased insulin dose estimates was guided by previous research, which showed that fill-based dose had a high correlation with self-reported insulin dose. ${ }^{26}$ In case of multiple basal and bolus insulin usage, the estimated TDD represented the sum of basal and bolus insulin.

Diabetes-related medication costs included actual planand patient-paid costs for all antihyperglycemic diabetes medications, including orals and injectables. Costs were not available for a portion of the pharmacy data; all cost analyses were therefore conducted on a subset of patients who had full cost data available. Costs were adjusted to 2016 price levels using Consumer Price Index information (medical care component) provided by the Bureau of Labor Statistics. ${ }^{27}$

\section{Patient Characteristics}

Baseline clinical conditions included the adapted Diabetes Complications Severity Index (aDCSI), ${ }^{28}$ the Quan-Charlson Comorbidity Index (QCI) ${ }^{29}$ and other targeted chronic comorbid conditions. Baseline oral diabetes medications, noninsulin diabetes injectables, and other common medications were identified using $\geq 1$ pharmacy claim (see Appendix A for codes).

\section{Statistical Analysis}

Adjustment for Selection Bias. A valid comparison of the V-Go and MDI cohorts requires similar baseline patient characteristics. Because this observational study design lacked treatment randomization, potential differences between cohorts that were capable of confounding the association between exposure and outcomes (leading to selection bias) were removed or reduced with propensity score matching. First, imbalances in baseline characteristics were determined using standardized mean differences (SMD), which measure differences in effect sizes across cohorts independent of sample size. $^{30}$ SMDs were calculated as the difference in means or proportions divided by the standard error; a larger SMD reflects larger effect sizes (or conversely, imbalances) in a given variable across 2 samples. An SMD>0.10 in absolute value was used to indicate potential imbalances across baseline patient characteristics. ${ }^{31}$ Subsequently, propensity scores were created via logistic regression including baseline insulin type, insulin fills, Alc level, oral diabetes therapy, noninsulin injectables, neuropathy, and dyslipidemia as independent variables. Then the V-Go and MDI cohorts were matched on the propensity scores using 1:1 nearest neighbor matching with calipers (equal to 0.2 units of the standard deviation [SD] of the logit of the propensity score) without replacement. ${ }^{31,32}$ Before assessing outcomes, the balance in baseline patient characteristics postmatching was again assessed via SMDs. ${ }^{31,33,34}$

Bivariate Analysis. For the assessment of glycemic control, means, SDs, and medians were reported at each time point as well as for the change from baseline to follow-up. Means were compared across cohorts using t-tests. The percentage of patients with Alc $\leq 9 \%$ was compared across cohorts using chi-square tests and using McNemar's test for the change over time within each cohort. For the assessment of insulin fills, TDD, and diabetes-related medication costs, means, SDs, and medians were reported at each time point as well as for the change from baseline to follow-up. Means were compared across cohorts using t-tests.

Multivariable Analysis. To evaluate robustness and adjust for baseline variables that remained imbalanced after matching, ordinary least squares regressions were used to evaluate the effect of V-Go versus MDI on the change from baseline in insulin fills, TDD, and diabetes-related medication costs (the list of variables included in the regression models can be found in the footnote to Table 4). Adjusted means (with 95\% confidence intervals) and difference-in-difference estimates (DID; calculated as [follow-up minus baseline for V-Go] minus [follow-up minus baseline for MDI], with 95\% confidence intervals and $P$ values) were reported.

\section{Sensitivity Analysis}

Insulin use and diabetes-related medication costs were also examined in a separate cohort that did not require the presence of Alc values during baseline or follow-up and hence included a larger sample. For this sensitivity analysis, a separate 1:1 propensity score match was conducted between V-Go and MDI patients to balance baseline characteristics.

\section{Results}

\section{Sample Identification}

As shown in Table 1, the overall prematch cohorts comprised 118 V-Go users and 3,018 MDI users for the main analysis, and 585 V-Go and 16,022 MDI users for the sensitivity analysis. 


\section{TABLE 1 Patient Selection for V-Go and MDI Cohorts}

\begin{tabular}{|c|c|c|c|}
\hline Step & Selection Criteria & V-Go Cohort, $n$ & MDI Cohort, $\mathrm{n}$ \\
\hline 1 & $\begin{array}{l}\text { Individuals aged 21-80 years with } \geq 1 \text { Rx claim of V-Go during January 1, 2012-April 30, } 2017 . \\
\text { Assign index date as the earliest claim for V-Go }\end{array}$ & 2,073 & - \\
\hline 1 & $\begin{array}{l}\text { Individuals aged 21-80 years with } \geq 2 \text { Rx claims of basal and } \geq 2 \text { Rx claims of bolus insulin within } \\
6 \text { months of each other between January } 1,2012 \text {, and April } 30,2017 \text {, and no V-Go fill anytime during } \\
\text { the study period. Assign index date as the earliest fill of bolus insulin }\end{array}$ & - & 71,007 \\
\hline 2 & Individuals with $\geq 6$ months pre-index continuous medical and pharmacy benefits coverage & 1,490 & 39,887 \\
\hline 3 & Individuals with $\geq 1$ medical claim for type 2 diabetes mellitus during baseline period & 1,433 & 35,395 \\
\hline 4 & Individuals with $\geq 1$ Rx claim for any insulin during baseline period (excluding index date) & 1,257 & - \\
\hline 4 & $\begin{array}{l}\text { Individuals with } \geq 1 \text { Rx claim for basal-bolus insulin, basal insulin only, or premixed insulin during } \\
\text { baseline period (excluding index date) }\end{array}$ & - & 22,855 \\
\hline 5 & $\begin{array}{l}\text { After exclusion of individuals with } \geq 1 \text { Rx claim for high-concentration insulin (U-500) during baseline } \\
\text { period (excluding index date) }\end{array}$ & 1,217 & 22,827 \\
\hline 6 & $\begin{array}{l}\text { After exclusion of individuals with } \geq 1 \text { Rx claim for only bolus insulin during baseline period } \\
\text { (excluding index date) }\end{array}$ & 1,070 & - \\
\hline 7 & After exclusion of individuals with $\geq 1$ medical or Rx claim for insulin pumps during study period & 968 & 22,725 \\
\hline 8 & $\begin{array}{l}\text { After exclusion of individuals with } \geq 1 \text { Rx claim for secondary diabetes, pregnancy or end-stage renal } \\
\text { failure during baseline period }\end{array}$ & 922 & 21,761 \\
\hline 9 & Main analysis (from step 8) & & \\
\hline $9 \mathrm{a}$ & Individuals with $\geq 1$ Alc value during baseline (index date -183 days to index date +15 days) & 218 & 4,547 \\
\hline $9 \mathrm{~b}$ & Individuals with $\geq 1$ Alc value at follow-up (index date +90 days to index date +410 days) & 118 & 3,018 \\
\hline $9 \mathrm{c}$ & Outcome 1: Glycemic control (matched pairs) & 118 & 118 \\
\hline $9 \mathrm{~d}$ & Outcome 2: Insulin use (matched pairs with $\geq 12$ months of post-index enrollment) & 74 & 74 \\
\hline $9 e$ & $\begin{array}{l}\text { Outcome 3: Diabetes-related medication cost (matched pairs with } \geq 12 \text { months of post-index enrollment } \\
\text { and full cost data availability) }\end{array}$ & 48 & 48 \\
\hline 10 & Sensitivity analysis (from step 8) & & \\
\hline $10 \mathrm{a}$ & Individuals with $\geq 12$ months of post-index enrollment & 585 & 16,022 \\
\hline $10 \mathrm{~b}$ & Outcome 2: Insulin use (matched pairs with $\geq 12$ months of post-index enrollment) & 585 & 585 \\
\hline $10 \mathrm{c}$ & $\begin{array}{l}\text { Outcome 3: Diabetes-related medication cost (matched pairs with } \geq 12 \text { months of post-index enrollment } \\
\text { and full cost data availability) }\end{array}$ & 464 & 464 \\
\hline
\end{tabular}

\section{Prematch Baseline Comparison}

The V-Go and MDI cohorts had similar mean age (56.5 vs. 56.3 years) and proportions of females (46.6\% vs. $42.3 \%$; Table 2). V-Go users were more likely to be enrolled in health maintenance organization plans (48.3\% vs. $31.9 \%)$ and live in the southern region (66.1\% vs. $43.4 \%)$, but a lower proportion was covered by preferred provider organization plans (34.7\% vs. 59.1\%), compared with MDI users. Similar baseline characteristics were observed for V-Go and MDI users in the sensitivity analysis cohort (Appendix B, available in online article). Although the V-Go and MDI cohorts had similar mean QCI and aDCSI scores, the V-Go group had a higher proportion of individuals with neuropathy (39.0\% vs. 25.0\%) and dyslipidemia ( $87.3 \%$ vs. $76.7 \%$ ) compared with MDI users pre-index. Metformin, sulfonylureas, and DPP-4 inhibitors were the most commonly used oral diabetes medications. Higher proportions of V-Go users received oral medications, specifically SGLT2 inhibitors, as well as GLP-1 receptor agonists (GLP-1 RAs), compared with MDI users. Pre-index, the V-Go cohort had the highest proportion of individuals with basal-bolus insulin (64.4\%), followed by basal insulin only (25.4\%), and premixed insulin only (10.2\%). In the MDI cohort, the largest proportion of individuals received basal insulin only (53.5\%) followed by basal-bolus insulin (41.0\%), and premixed insulin only (5.5\%).

\section{Postmatch Baseline Comparison}

The matched cohorts included 118 pairs for the main analysis and 585 pairs for the sensitivity analysis. In the main analysis, V-Go and MDI users were balanced on key baseline characteristics including previous insulin type, number of insulin fills, age, gender, overall oral diabetes medication use and GLP-1 RA use (SMD $\leq 0.1)$. Differences remained for health plan type, region of residence, treating physician specialty, and some comorbidities and medications (SMD>0.1; Table 2). Results were similar in the sensitivity analysis cohort (Appendix B).

\section{Glycemic Control}

Before matching, mean (SD) baseline Alc values were higher for V-Go versus MDI users (9.3 [1.56] vs. 9.0 [1.87], SMD 0.13; medians 9.1 vs. 8.7). After matching, Alc values were similar 
Effect of V-Go Versus Multiple Daily Injections on Glycemic Control, Insulin Use, and Diabetes Medication Costs Among Individuals with Type 2 Diabetes Mellitus

TABLE 2 Baseline Demographic and Clinical Characteristics and Medication Use Among V-Go and MDI Users: Before and After Matching for Main Analysis

\begin{tabular}{|c|c|c|c|c|c|}
\hline \multirow[b]{2}{*}{ Baseline Characteristics } & \multicolumn{3}{|c|}{ Cohorts Before Matching } & \multicolumn{2}{|c|}{ Cohorts After Matchinga } \\
\hline & V-Go Cohort & MDI Cohort & $\mathrm{SMD}^{\mathrm{b}}$ & MDI Cohort & SMD \\
\hline Sample size & 118 & 3,018 & & 118 & \\
\hline \multicolumn{6}{|l|}{ Demographic characteristics } \\
\hline Female, n (\%) & $55 \quad(46.6)$ & $1,278 \quad(42.3)$ & 0.09 & $57 \quad(48.3)$ & -0.03 \\
\hline Age at index, in years, mean (SD), median & $56.5(9.08), 58.0$ & $56.3(10.08), 57.0$ & 0.02 & $56.9(9.46), 57.0$ & -0.05 \\
\hline HMO plan, n (\%) & $57 \quad(48.3)$ & $962 \quad(31.9)$ & 0.34 & $35 \quad(29.7)$ & 0.39 \\
\hline PPO plan, n (\%) & $41 \quad(34.7)$ & $1,785 \quad(59.1)$ & -0.50 & $72 \quad(61.0)$ & -0.55 \\
\hline CDHP plan, n (\%) & $20 \quad(16.9)$ & $(9.0)$ & 0.24 & $(9.3)$ & 0.23 \\
\hline Commercial insurance, $\mathrm{n}(\%)$ & $112 \quad(94.9)$ & $2,705 \quad(89.6)$ & 0.20 & $100 \quad(84.7)^{\mathrm{c}}$ & 0.34 \\
\hline \multicolumn{6}{|l|}{ Region, n (\%) } \\
\hline Northeast & $22 \quad(18.6)$ & $(17.2)$ & 0.04 & $24 \quad(20.3)$ & -0.04 \\
\hline Midwest/West & $18 \quad(15.3)$ & $1,187 \quad(39.3)^{\mathrm{c}}$ & -0.56 & $48 \quad(40.7)^{c}$ & -0.59 \\
\hline South & $78 \quad(66.1)$ & $1,311 \quad(43.4)^{\mathrm{c}}$ & 0.47 & $46 \quad(39.0)^{c}$ & 0.56 \\
\hline \multicolumn{6}{|l|}{ Physician specialty, ${ }^{\mathrm{d}} \mathrm{n}(\%)$} \\
\hline Endocrinologist & $42 \quad(35.6)$ & $1,073 \quad(35.6)$ & 0.00 & $43 \quad(36.4)$ & -0.02 \\
\hline PCP & $32 \quad(27.1)$ & $1,322 \quad(43.8)^{\mathrm{c}}$ & -0.35 & $42 \quad(35.6)^{c}$ & -0.18 \\
\hline Other & $(5.1)$ & $(8.1)^{\mathrm{c}}$ & -0.12 & $13 \quad(11.0)^{\mathrm{c}}$ & -0.22 \\
\hline Missing/unknown & $38 \quad(32.2)$ & $380 \quad(12.6)^{\mathrm{c}}$ & 0.48 & $20 \quad(16.9)^{c}$ & 0.36 \\
\hline \multicolumn{6}{|l|}{ Clinical conditions of interest } \\
\hline QCI score, mean (SD), median & $2.2(1.53), 2.0$ & $2.3(1.73), 2.0$ & -0.06 & $2.4(1.91), 2.0$ & -0.14 \\
\hline aDCSI score, mean (SD), median & $1.5(1.94), 1.0$ & $1.5(1.80), 1.0$ & 0.02 & $1.92(2.02), 1.0$ & -0.21 \\
\hline \multicolumn{6}{|l|}{ Diabetic complications, n (\%) } \\
\hline Retinopathy & $24 \quad(20.3)$ & $485 \quad(16.1)$ & 0.11 & $16 \quad(13.6)$ & 0.18 \\
\hline Neuropathy & $\begin{array}{ll}46 & (39.0) \\
\end{array}$ & $753 \quad(25.0)^{c}$ & 0.30 & $45 \quad(38.1)$ & 0.02 \\
\hline Nephropathy & $23 \quad(19.5)$ & $550 \quad(18.2)$ & 0.03 & $23 \quad(19.5)$ & 0.00 \\
\hline Cerebrovascular & $(5.1)$ & $(5.0)$ & 0.00 & $11 \quad(9.3)$ & -0.16 \\
\hline Cardiovascular & $24 \quad(20.3)$ & $744 \quad(24.7)$ & -0.10 & $37 \quad(31.4)$ & -0.25 \\
\hline Peripheral vascular disease & $(9.3)$ & $(10.8)$ & -0.05 & $17 \quad(14.4)$ & -0.16 \\
\hline \multicolumn{6}{|l|}{ Other comorbidities, n (\%) } \\
\hline Hypertension & $94 \quad(79.7)$ & $2,355 \quad(78.0)$ & 0.04 & $106 \quad(89.8)^{\mathrm{c}}$ & -0.29 \\
\hline Dyslipidemia & $103 \quad(87.3)$ & $2,314 \quad(76.7)^{\mathrm{c}}$ & 0.28 & $103 \quad(87.3)$ & 0.00 \\
\hline Mental illness & $20 \quad(16.9)$ & $553 \quad(18.3)$ & -0.04 & $28 \quad(23.7)$ & -0.17 \\
\hline
\end{tabular}

at baseline (9.3 [1.56] vs. 9.2 [1.47], SMD 0.06; medians 9.2 vs. 9.0) and also at the end of follow-up (8.6 [1.69] vs. 8.8 [1.64], $P=0.514$; Table 3). In addition, there was no significant difference in the change in mean Alc values from baseline between V-Go and MDI users (-0.6 [1.59] vs. -0.4 [1.61], $P=0.263$; DID $=-0.2$, $P=0.378)$. Both cohorts had improvements in glycemic control during follow-up relative to baseline (\% with Alc $\leq 9 \%$, pre/ post: V-Go 49/69, $P<0.001$; MDI 50/60, $P=0.046$ ). There was no statistically significant difference between the 2 cohorts in Alc control during the follow-up period $(P=0.134)$.

\section{Insulin Use}

While both cohorts had similar insulin prescription ( $\mathrm{Rx}$ ) fills and insulin TDD at baseline, V-Go users had fewer insulin Rx fills (mean change: -0.8 vs. +1.8 fills, $P<0.001 ;-17 \%$ vs. $+38 \%$ ) and a decline in TDD (mean change: -29.0 vs. +5.8 units per day,
$P<0.001 ;-21 \%$ vs. $+4 \%)$ compared with MDI users during the last 6 months of follow-up (Table 3). In the sensitivity analysis cohort, V-Go users also had fewer insulin Rx fills (mean change from baseline: -0.4 vs. +1.8 fills, $P<0.001$ ), and a decline in TDD (mean change: -24.2 vs. +6.0 units per day, $P<0.001$ ) compared with MDI users. When adjusting for imbalanced covariates postmatching via regression analyses, the direction and magnitude of the association remained consistent: V-Go users had fewer insulin Rx fills $(P<0.001)$ and a decrease in insulin TDD $(P<0.001)$ compared with MDI users during the final 6 months of follow-up (Table 4).

\section{Diabetes-Related Medication Costs}

While V-Go and MDI users had similar baseline diabetesrelated medication costs in both the main $(n=48)$ and sensitivity analysis $(n=464)$ cohorts, V-Go users experienced 
Effect of V-Go Versus Multiple Daily Injections on Glycemic Control, Insulin Use, and Diabetes Medication Costs Among Individuals with Type 2 Diabetes Mellitus

TABLE 2 Baseline Demographic and Clinical Characteristics and Medication Use Among V-Go and MDI Users: Before and After Matching for Main Analysis (continued)

\begin{tabular}{|c|c|c|c|c|c|}
\hline \multirow[b]{2}{*}{ Baseline Characteristics } & \multicolumn{3}{|c|}{ Cohorts Before Matching } & \multicolumn{2}{|c|}{ Cohorts After Matchinga } \\
\hline & V-Go Cohort & MDI Cohort & $\mathrm{SMD}^{\mathrm{b}}$ & MDI Cohort & SMD \\
\hline \multicolumn{6}{|l|}{ Insulin use } \\
\hline \multicolumn{6}{|c|}{ Previous insulin exposure, $\mathrm{n}(\%)$} \\
\hline Basal-bolus insulin & $76 \quad(64.4)$ & $1,238 \quad(41.0)^{c}$ & 0.48 & $75 \quad(63.6)$ & 0.02 \\
\hline Basal insulin only & $30 \quad(25.4)$ & $1,614 \quad(53.5)^{\mathrm{c}}$ & -0.60 & $31 \quad(26.3)$ & -0.02 \\
\hline Premixed insulin only & $12 \quad(10.2)$ & $(5.5)^{c}$ & 0.17 & $12 \quad(10.2)$ & 0.00 \\
\hline \multicolumn{6}{|c|}{ Fills of insulin, mean (SD), median } \\
\hline Basal insulin & $2.6(1.88), 2.0$ & $4.0(2.13), 4.0^{c}$ & -0.68 & $2.7(1.70), 2.0$ & -0.06 \\
\hline Bolus insulin & $1.7(1.65), 1.0$ & $1.2(1.84), 0.0^{c}$ & 0.26 & $1.6(1.64), 1.0$ & 0.05 \\
\hline Premixed insulin & & $0.3(1.18), 0.0$ & 0.07 & $0.4(1.19), 0.0$ & -0.01 \\
\hline \multicolumn{6}{|l|}{ Baseline other medications } \\
\hline \multicolumn{6}{|l|}{ Oral diabetes therapy, $\mathrm{n}(\%)$} \\
\hline Biguanides & $62 \quad(52.5)$ & $(47.3)$ & 0.11 & $71 \quad(60.2)$ & -0.15 \\
\hline Sulfonylureas & $27 \quad(22.9)$ & $(25.1)$ & -0.05 & $37 \quad(31.4)$ & -0.19 \\
\hline DPP-4i & $22 \quad(18.6)$ & $(16.5)$ & 0.06 & $20 \quad(16.9)$ & 0.04 \\
\hline SGLT2i & $15 \quad(12.7)$ & $(3.3)^{\mathrm{c}}$ & 0.35 & $5 \quad(4.2)^{c}$ & 0.31 \\
\hline TZD & $8 \quad(6.8)$ & $(5.1)$ & 0.07 & $(9.3)$ & -0.09 \\
\hline Any of the above & $89 \quad(75.4)$ & $1,830 \quad(60.6)^{c}$ & 0.32 & $89 \quad(75.4)$ & 0.00 \\
\hline \multicolumn{6}{|c|}{ Injectable noninsulin therapy, n (\%) } \\
\hline GLP-1 receptor agonists & $26 \quad(22.0)$ & $(11.4)^{c}$ & 0.29 & $26 \quad(22.0)$ & 0.00 \\
\hline \multicolumn{6}{|l|}{ Other medications, n (\%) } \\
\hline Antihypertensives & $97 \quad(82.2)$ & $2,501 \quad(82.9)$ & -0.02 & $103 \quad(87.3)$ & -0.14 \\
\hline Antihyperlipidemics & $90 \quad(76.3)$ & $2,152 \quad(71.3)$ & 0.11 & $94 \quad(79.7)$ & -0.08 \\
\hline CVD medications $^{\mathrm{e}}$ & $90 \quad(76.3)$ & $2,044 \quad(67.7)$ & 0.19 & $88 \quad(74.6)$ & 0.04 \\
\hline \multicolumn{6}{|c|}{ 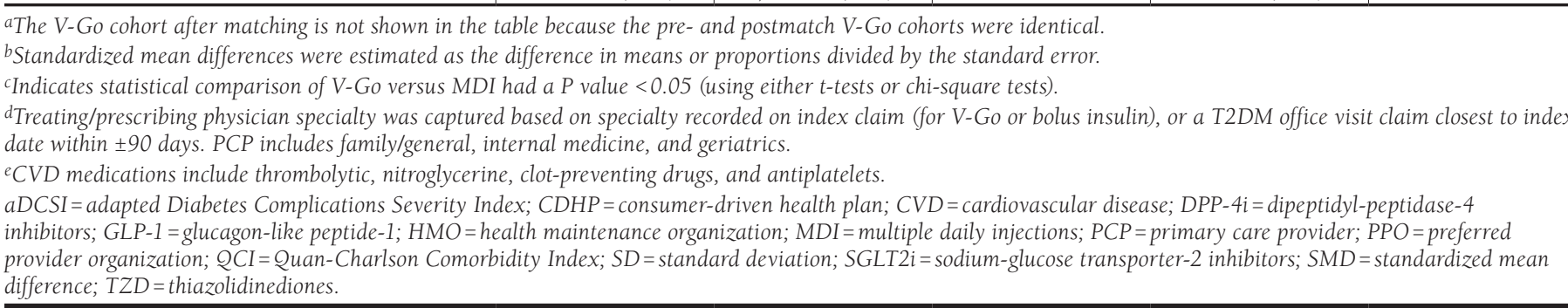 } \\
\hline
\end{tabular}

a lower increase in diabetes-related medication costs (mean change in 2016 USD: \$341 vs. \$1,628, $P=0.012$; $+10 \%$ vs. $+47 \%$ ) versus MDI users during the last 6 months of follow-up (Table 3). The sensitivity analysis cohort had a similar pattern suggesting a lower increase in diabetesrelated medication costs (mean change in 2016 USD: \$506 vs. $\$ 1,129, P<0.001)$ for $\mathrm{V}$-Go users. Results of the regression analysis of costs were consistent with this finding (Table 4).

\section{Discussion}

In this study, both in the main and sensitivity analyses, the number of insulin fills and insulin dosing at follow-up were significantly lower among V-Go users relative to MDI therapy users. As a consequence, the increase in diabetesrelated medication costs was smaller for V-Go users compared with MDI users during the last 6 months of follow-up. Regression analysis showed that adjusting for patient characteristics which were not balanced after matching led to similar outcomes. This is particularly important as V-Go users (who represent a new technology) were indeed different from MDI users along several dimensions at baseline (region of residence, some comorbidities, and Alc).

To our knowledge, this is the first administrative claimsbased study that assessed glycemic control, insulin use (prescription fills and TDD) and costs for these cohorts for up to a year. V-Go users had a mean decrease in Alc of $0.6 \%$ from baseline versus $0.4 \%$ for MDI; the improvements were statistically significant within each cohort, but the difference across cohorts was not. It is instructive to put these results, as well as our findings on insulin use and costs, into context with previous research. A handful of shorter-term studies, most with much smaller sample sizes, reported similar characteristics 
Effect of V-Go Versus Multiple Daily Injections on Glycemic Control, Insulin Use, and Diabetes Medication Costs Among Individuals with Type 2 Diabetes Mellitus

TABLE 3 Descriptive Results for Baseline and Follow-Up A1c Levels, Insulin Fills, Total Daily Dose of Insulin, and Diabetes-Related Medication Cost Among V-Go and MDI Users

\begin{tabular}{|c|c|c|c|c|c|c|c|c|c|c|c|c|}
\hline & \multicolumn{7}{|c|}{ Main Analysis Cohort } & \multicolumn{5}{|c|}{ Sensitivity Analysis Cohort } \\
\hline \multicolumn{13}{|c|}{ Alc Levels } \\
\hline & \multicolumn{3}{|c|}{$\begin{array}{c}\text { V-Go } \\
(n=118)\end{array}$} & \multicolumn{3}{|c|}{$\begin{array}{c}\text { MDI } \\
(\mathrm{n}=118)\end{array}$} & & & & & & \\
\hline & \multicolumn{2}{|c|}{ Mean (SD) } & \multirow{2}{*}{$\begin{array}{c}\text { Median } \\
9.2\end{array}$} & \multicolumn{2}{|c|}{ Mean (SD) } & \multirow{2}{*}{$\begin{array}{c}\text { Median } \\
9.0\end{array}$} & & & & & & \\
\hline Baseline (6m) & 9.3 & (1.6) & & 9.2 & (1.5) & & $\frac{P \text { Value }}{0.640}$ & & & & & \\
\hline Follow-up (3-13m) & 8.6 & $(1.7)$ & 8.4 & 8.8 & (1.6) & 8.3 & 0.514 & & & & & \\
\hline Change & -0.6 & $(1.5)$ & -0.6 & -0.4 & $(1.6)$ & -0.4 & 0.263 & & & & & \\
\hline \multicolumn{13}{|c|}{ Number of Insulin Fills } \\
\hline & \multicolumn{3}{|c|}{$\begin{array}{l}\text { V-Go } \\
(\mathrm{n}=74)\end{array}$} & \multicolumn{3}{|c|}{$\begin{array}{c}\text { MDI } \\
(\mathbf{n}=74)\end{array}$} & \multirow[b]{2}{*}{$P$ Value } & \multicolumn{2}{|c|}{$\begin{array}{c}\text { V-Go } \\
(\mathrm{n}=585)\end{array}$} & \multicolumn{2}{|c|}{$\begin{array}{c}\text { MDI } \\
(n=585)\end{array}$} & \multirow[b]{2}{*}{$P$ Value } \\
\hline & \multicolumn{2}{|c|}{ Mean (SD) } & Median & \multicolumn{2}{|c|}{ Mean (SD) } & Median & & Mean (SD) & \multirow{2}{*}{$\begin{array}{c}\text { Median } \\
4.0\end{array}$} & Mean (SD) & \multirow{2}{*}{$\frac{\text { Median }}{4.0}$} & \\
\hline Baseline (6m) & 4.7 & $(2.6)$ & 4.5 & 4.8 & $(2.6)$ & 4.0 & 0.784 & $4.6 \quad(2.8)$ & & $(2.6)$ & & 0.587 \\
\hline Follow-up (L:6m) & 3.9 & $(2.3)$ & 4.0 & 6.6 & (3.3) & 6.0 & 0.003 & $4.2 \quad(2.8)$ & 4.0 & $(3.4)$ & 6.0 & $<0.001$ \\
\hline Change & -0.8 & $(3.1)$ & -1.0 & 1.8 & $(3.0)$ & 1.0 & $<0.001$ & $(3.1)$ & 0.0 & $(3.1)$ & 2.0 & $<0.001$ \\
\hline \multicolumn{13}{|c|}{ Insulin Total Daily Dose, Units per Day } \\
\hline & \multicolumn{3}{|c|}{$\begin{array}{l}\text { V-Go } \\
(\mathrm{n}=74)\end{array}$} & \multicolumn{3}{|c|}{$\begin{array}{c}\text { MDI } \\
(\mathrm{n}=74)\end{array}$} & & \multicolumn{2}{|c|}{$\begin{array}{c}\text { V-Go } \\
(\mathrm{n}=585)\end{array}$} & \multicolumn{2}{|c|}{$\begin{array}{c}\text { MDI } \\
(\mathrm{n}=585)\end{array}$} & \\
\hline & \multicolumn{2}{|c|}{ Mean (SD) } & Median & Mean & (SD) & Median & $P$ Value & Mean (SD) & Median & Mean (SD) & Median & $P$ Value \\
\hline Baseline (6m) & 141.0 & $(62.1)$ & 133.3 & 132.3 & $(56.4)$ & 108.1 & 0.251 & $136.6 \quad(67.0)$ & 126.9 & $142.5 \quad(75.3)$ & 116.7 & 0.981 \\
\hline Follow-up (L:6m) & 112.0 & $(51.1)$ & 100.0 & 138.1 & $(69.2)$ & 113.3 & 0.008 & $112.4 \quad(57.9)$ & 100.0 & $148.5(78.8)$ & 120.5 & $<0.001$ \\
\hline Change & -29.0 & $(61.1)$ & -33.3 & 5.8 & $(57.5)$ & 0.0 & $<0.001$ & $-24.2 \quad(69.7)$ & -26.9 & $6.0 \quad(59.3)$ & 0.0 & $<0.001$ \\
\hline & & & & Dial & etes-Re & ated Med & ation Cos & 2016 USD $^{a}$ & & & & \\
\hline & & $\begin{array}{l}\text { V-Go } \\
(\mathrm{n}=48)\end{array}$ & & & $\begin{array}{r}\text { MDI } \\
(\mathbf{n}=48\end{array}$ & & & $\begin{array}{r}\text { V-Go } \\
(\mathrm{n}=46\end{array}$ & & $\begin{array}{r}\text { MDI } \\
(\mathrm{n}=46\end{array}$ & & \\
\hline & Mear & n (SD) & Median & Mean & (SD) & Median & $P$ Value & Mean (SD) & Median & Mean (SD) & Median & $P$ Value \\
\hline Baseline $(6 \mathrm{~m})$ & 3,526 & $(2,582)$ & 2,658 & 3,500 & $(4,036)$ & 2,658 & 0.962 & $3,431(2,467)$ & 2,778 & $3,260(2,244)$ & 2,838 & 0.267 \\
\hline Follow-up (L:6m) & 3,867 & $(2,799)$ & 2,908 & 5,128 & $(5,247)$ & 4,286 & 0.107 & $3,937(3,267)$ & 3,139 & $4,389(2,964)$ & 3,899 & 0.073 \\
\hline Change & 341 & $(2,330)$ & 470 & 1,628 & $(2,594)$ & 949 & 0.012 & $506(2,849)$ & 389 & $1,129(2,214)$ & 900 & $<0.001$ \\
\hline
\end{tabular}

a Among subgroup of patients with complete cost data.

Alc=hemoglobin Alc; L:6m=last 6 months of the 12-month follow-up period; $m=$ months; MDI= multiple daily injections; SD=standard deviation; USD=U.S. dollars.

for V-Go and MDI users as our study on age, baseline diabetes complications, and previous insulin treatment among individuals with T2DM. ${ }^{17,21-24}$ In a small $(\mathrm{N}=23)$ 24-week telephone survey study, Rosenfeld et al. (2012) reported that V-Go users had a mean decrease in Alc of $1.2 \%$ after 12 weeks of treatment; the mean level increased by $0.6 \%$ after V-Go treatment was discontinued. ${ }^{23}$

Several other previous studies used EMR datasets with larger sample sizes. Sutton et al. reported significant Alc reductions, a significant decrease in TDD, and reduced pharmacy costs for a sample of $\mathrm{N}=103 \mathrm{~V}$-Go users. ${ }^{18}$ For the 58 patients with MDI at baseline, the mean (SE) reduction was $1.5 \%(0.31)$ in Alc and 29 units per day (5.0) in TDD after 14 months, with a decrease in overall costs for diabetes medications of $\$ 25.00$ per patient per month. Everitt et al. compared 108 patients (85 V-Go users, 23 conventional insulin delivery users [CID]) who were persistent to therapy for $\geq 5$ months; V-Go patients experienced significant reductions in Alc (-1.4\% vs. $-0.2 \%)$ and TDD (-4.2 vs. 13.2 units per day) compared with CID. ${ }^{19}$ Total therapy costs increased by similar amounts in both cohorts (V-Go \$162, CID \$168), but because of the Alc improvements seen for $\mathrm{V}-\mathrm{Go}$, the cost per $1 \%$ drop in Alc was much lower in V-Go cohort (\$109 vs. \$841). Also using EMR data, Lajara et al. (2015) evaluated 204 patients with suboptimal Alc > 7\% (88\% on insulin) who switched to V-Go. ${ }^{21}$ The authors reported significant decreases in Alc levels at weeks 14 and 27 follow-up. Similar to our findings, patients using V-Go in this study needed significantly less insulin (a drop to 58 units per day at week 27 versus 86-99 units per day at baseline, $P<0.001$ ). In a subsequent study to evaluate economic impact (using a subgroup of $\mathrm{n}=97$ with Alc $>9 \%$ at baseline), Lajara et al. (2016) reported that after approximately 14 weeks of treatment with V-Go, patients had a decrease of $2 \%$ from their baseline Alc level of $10.5 \%$ and a reduction in diabetes-related pharmacy costs (\$119 per patient per month). ${ }^{22}$

Finally, in a pragmatic trial, Cziraky et al. (2019) randomized 169 patients to use V-Go and 246 to receive standard treatment optimization (STO; i.e., treatment according to 
Effect of V-Go Versus Multiple Daily Injections on Glycemic Control, Insulin Use, and Diabetes Medication Costs Among Individuals with Type 2 Diabetes Mellitus

TABLE 4 Regression Results for Change in A1c Levels, Insulin Fills, Total Daily Dose of Insulin, and Diabetes-Related Medication Cost Among V-Go and MDI Users

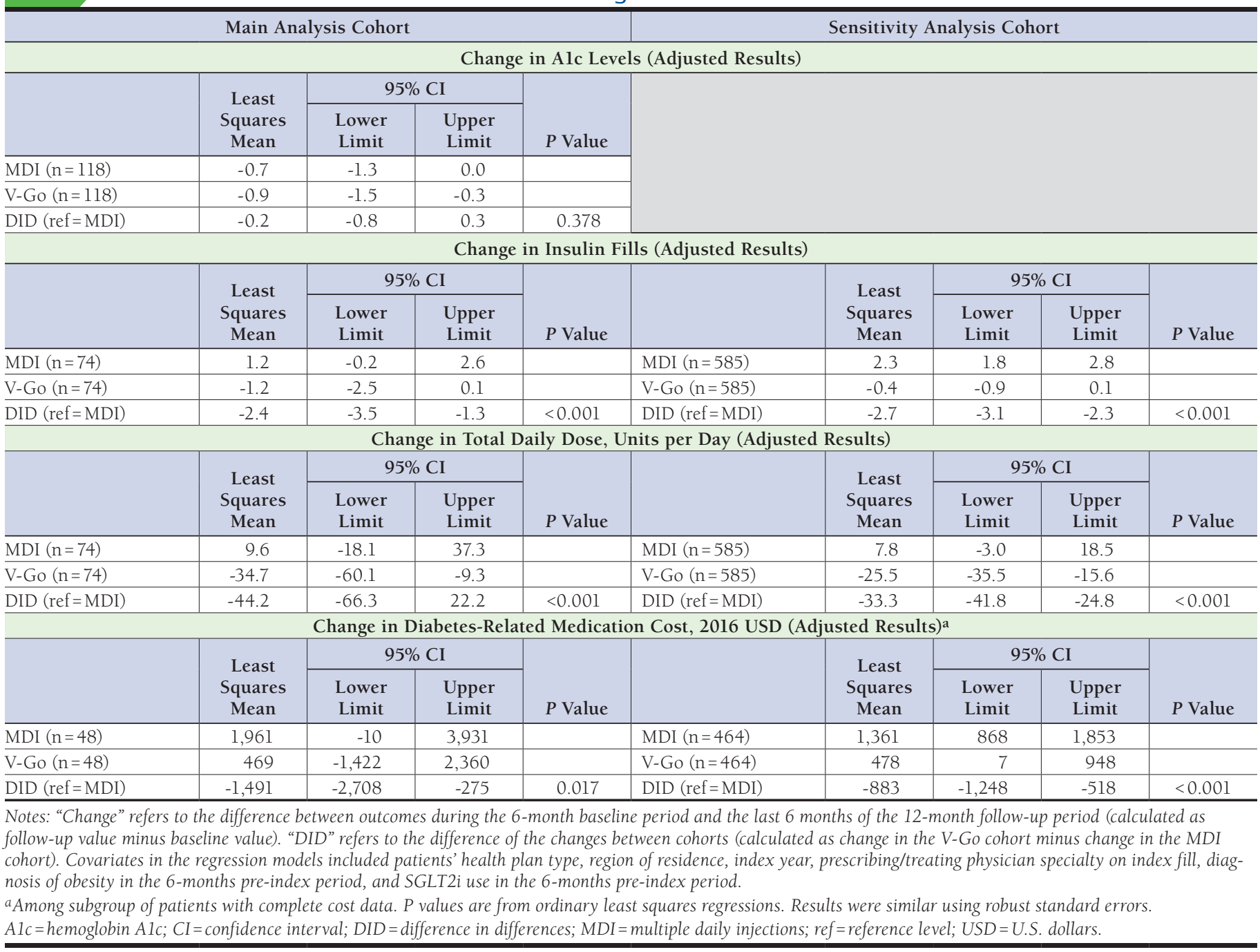

routine clinical practice). ${ }^{17}$ Of those, 208 patients (95 V-Go, 113 STO) were on MDI. Starting from Alc baseline levels of 9.9\% for V-Go users and 9.4\% for MDI users, Alc levels of V-Go users decreased by $1.0 \%$ versus $0.4 \%$ for MDI users at the end of the study. As in our study, V-Go users had a significant decrease in TDD (from 75 to 55 units per day), while MDI patients did not ( 77 units per day throughout). The mean cost of diabetes treatment per patient per day was lower for V-Go (\$31) versus MDI (\$32) groups. Because of the larger decrease in Alc, the difference between the cohorts in costs per 1\% drop in Alc was larger than the difference in mean treatment costs (V-Go \$24 vs. MDI \$59).

Compared with these previous findings, our study used a different design while leading to mostly consistent results. In particular, our longer-term comparative design also showed that V-Go is associated with reductions in insulin use and cost savings, as previous shorter-term studies had done. So far, our study is unique in also looking at glycemic control over a longer time horizon; unlike shorter-term studies, the gain in Alc control was not statistically superior to the one seen for MDI patients. Several factors may play a role here, including adherence (which this study did not evaluate), sample selection (e.g., we included all patients starting from 2012 when V-Go was launched and their characteristics and outcomes may be different from those who started later when physicians were more accustomed to the new device), and statistical power (availability of Alc results was limited in our study).

Overall, these results highlight issues with MDI treatment, notably the inability to optimize TDD and contain costs. In general, individuals on insulin with uncontrolled Alc are often 
treated aggressively by intensification of basal insulin. 2,35,36 An estimated $82 \%$ also need intensification that incorporates mealtime bolus within 3 years of insulin initiation. ${ }^{37}$ Such intensification and other treatment complications have been linked to poor adherence and negative patient perceptions regarding insulin therapy. ${ }^{38-41}$ Inefficiencies in insulin therapy appear evident, which suggests a role for alternative modalities including more convenient and consistent delivery systems. ${ }^{17,21,22,24,42}$ The results of our study add value to the body of literature available to patients, clinicians, and payers who are engaged with treatment and economic decisions associated with T2DM management. Our results indicate that V-Go may provide an effective alternative to improve glycemic control with lower cost of diabetes medications for individuals with T2DM requiring basal or basal-bolus regimens.

\section{Limitations}

This study has some limitations. Sample size for the primary objective on glycemic control was limited (for the secondary objectives, we performed sensitivity analysis on a larger cohort, which yielded consistent results). We conducted intent-to-treat analysis; we did not examine treatment adherence/persistence, and it is possible that patients had discontinued use of V-Go/ MDI or insulin at the time that some follow-up outcomes were measured. While we carefully balanced observable confounders through matching, selection bias on unmeasured confounders may remain, primarily because of missing information on clinical characteristics (e.g., patient/physician preferences, weight, duration of diabetes) and the socioeconomic status of patients. This study did not evaluate outcomes during the first 6 months after treatment initiation (the transition period), since our focus was on longer-term outcomes.

Our findings were also subject to typical claims-based limitations such as coding errors, and the inability to determine whether prescription fills were actually taken by patients. Furthermore, the estimated insulin TDD may not accurately reflect the self-administered TDD. Previous research, however, showed that fill-based TDD had high correlation with selfreported/administered TDD, and claims-based estimated TDD tended to be only slightly higher than the actual dose. ${ }^{26}$

Study findings are applicable to individuals with T2DM enrolled in commercial or Medicare Advantage health plans and may not be generalizable to T2DM populations in general. Cost data were available for a subset of patients and generalization of cost results may be limited.

\section{Conclusions}

This study was the first to report on clinical and economic outcomes associated with the use of the V-Go insulin delivery device over a follow-up period of up to 1 year. V-Go users had similar glycemic control but lower insulin use and lower diabetes medication costs during follow-up compared with
MDI users. Antidiabetic therapy with V-Go represents an opportunity to improve quality measures more efficiently and at a lower cost in a population with T2DM requiring basalbolus therapy.

\section{Authors}

AMIT D. RAVAL, PhD; SITING ZHOU, PhD; MICHAEL GRABNER, PhD; JOHN BARRON, PharmD; and RALPH QUIMBO, MA, HealthCore, Wilmington, Delaware. MATTHEW H. NGUYEN, PharmD, Valeritas, Bridgewater, New Jersey.

AUTHOR CORRESPONDENCE: Siting Zhou, PhD, Senior Researcher, HealthCore, 123 Justison St., Ste. 200, Wilmington, DE 19801. Tel.: 302.230.2000; E-mail: szhou@healthcore.com.

\section{DISCLOSURES}

This study was funded by Valeritas. Nguyen is an employee of Valeritas. Zhou, Grabner, Barron, and Quimbo are employees of HealthCore, which received funding for this study from Valeritas. Raval was an employee of HealthCore at the time the study was conducted.

Partial findings from this study were presented at the International Society of Pharmacoeconomics and Outcomes Research 23rd Annual International Meeting; May 19-23, 2018; Baltimore, MD; and the 54th European Association for the Study of Diabetes Annual Meeting; October 1-5, 2018; Berlin, Germany.

\section{ACKNOWLEDGMENTS}

The authors thank Bernard Tulsi for writing and editorial support on this manuscript.

\section{REFERENCES}

1. Professional Practice Committee: Standards of Medical Care in Diabetes-2018. Diabetes Care. 2018;41(Suppl 1):S3.

2. Dalal MR, Grabner M, Bonine N, Stephenson JJ, DiGenio A, Bieszk N. Are patients on basal insulin attaining glycemic targets? Characteristics and goal achievement of patients with type 2 diabetes mellitus treated with basal insulin and physician-perceived barriers to achieving glycemic targets. Diabetes Res Clin Pract. 2016;121:17-26.

3. Raccah D. Basal insulin treatment intensification in patients with type 2 diabetes mellitus: a comprehensive systematic review of current options. Diabetes Metab. 2017:43(2):110-24.

4. Raccah D, Huet D, Dib A, et al. Review of basal-plus insulin regimen options for simpler insulin intensification in people with type 2 diabetes mellitus. Diabet Med. 2017;34(9):1193-204.

5. Turner RC, Cull CA, Frighi V, Holman RR. Glycemic control with diet, sulfonylurea, metformin, or insulin in patients with type 2 diabetes mellitus: progressive requirement for multiple therapies (UKPDS 49). UK Prospective Diabetes Study (UKPDS) Group. JAMA. 1999;281(21):2005-12.

6. Farsaei S, Radfar M, Heydari Z, Abbasi F, Qorbani M. Insulin adherence in patients with diabetes: risk factors for injection omission. Prim Care Diabetes. 2014;8(4):338-45.

7. Gherman A, Schnur J, Montgomery G, Sassu R, Veresiu I, David D. How are adherent people more likely to think? A meta-analysis of health beliefs and diabetes self-care. Diabetes Educ. 2011;37(3):392-408. 
8. Sarbacker GB, Urteaga EM. Adherence to insulin therapy. Diabetes Spectr. 2016;29(3):166-70

9. Consensus Development Conference on Insulin Resistance. 5-6 November 1997. American Diabetes Association. Diabetes Care. 1998;21(2):310-14.

10. Church TJ, Haines ST. Treatment approach to patients with severe insulin resistance. Clin Diabetes. 2016;34(2):97-104.

11. Crasto W, Jarvis J, Hackett E, et al. Insulin U-500 in severe insulin resistance in type 2 diabetes mellitus. Postgrad Med J. 2009;85(1002):219-22.

12. Dall T, Thiselton D, Varvel S. Targeting insulin resistance: the ongoing paradigm shift in diabetes prevention. Am J Manag Care. 2013;19(Spec No. 2):E7.

13. O'Brien JA, Patrick AR, Caro J. Estimates of direct medical costs for microvascular and macrovascular complications resulting from type 2 diabetes mellitus in the United States in 2000. Clin Ther. 2003;25(3):1017-38.

14. Sherman BW. Novel devices for delivering diabetes medications: their value and some coverage considerations. Manag Care. 2017;26(3):42-45.

15. Zullig LL, Gellad WF, Moaddeb J, et al. Improving diabetes medication adherence: successful, scalable interventions. Patient Prefer Adherence. 2015;9:139-49.

16. Valeritas. V-Go instructions for patient use. ART-037 Rev H. September 2011. Available at: https://www.go-vgo.com/sites/default/files/upload/ instructions-for-patient-use.pdf. Accessed May 30, 2019.

17. Cziraky MJ, Abbott S, Nguyen M, et al. A pragmatic clinical trial to compare the real-world effectiveness of V-Go versus standard delivery of insulin in patients with advanced type 2 diabetes. JHEOR. 2019;6(2):70-83.

18. Sutton D, Higdon CD, Nikkel C, Hilsinger KA. Clinical benefits over time associated with use of V-Go wearable insulin delivery device in adult patients with diabetes: a retrospective analysis. Adv Ther. 2018;35(5):631-43.

19. Everitt B, Harrison HC Jr, Nikkel C, Laswell E, Chen AMH. Clinical and economic considerations based on persistency with a novel insulin delivery device versus conventional insulin delivery in patients with type 2 diabetes: a retrospective analysis. Res Social Adm Pharm. September 26, 2018 [Epub ahead of print]. Available at: https://www.sciencedirect.com/science/ article/pii/S1551741118306442?via\%3Dihub. Accessed June 19, 2019.

20. Johns BR, Jones TC, Sink JH 2nd, Cooke CE. Real-world assessment of glycemic control after V-Go initiation in an endocrine practice in the southeastern United States. J Diabetes Sci Technol. 2014;8(5):1060-61.

21. Lajara R, Fetchick DA, Morris TL, Nikkel C. Use of V-Go insulin delivery device in patients with sub-optimally controlled diabetes mellitus: a retrospective analysis from a large specialized diabetes system. Diabetes Ther. 2015;6(4):531-45

22. Lajara R, Nikkel C, Abbott S. The clinical and economic impact of the V-Go disposable insulin delivery device for insulin delivery in patients with poorly controlled diabetes at high risk. Drugs Real World Outcomes. 2016:3(2):191-99.

23. Rosenfeld CR, Bohannon NJ, Bode B, et al. The V-Go insulin delivery device used in clinical practice: patient perception and retrospective analysis of glycemic control. Endocr Pract. 2012;18(5):660-67.

24. Winter A, Lintner M, Knezevich E. V-Go insulin delivery system versus multiple daily insulin injections for patients with uncontrolled type 2 diabetes mellitus. J Diabetes Sci Technol. 2015;9(5):1111-16.

25. National Committee for Quality Assurance. Health Effectiveness Data and Information Set (HEDIS) Volume 2: Technical Specifications for Health Plans. 2017. Available at: https://www.ncqa.org/hedis/measures/. Accessed June 9, 2019
26. Stephenson J, Raval A, Kern D, Bae J. E3-Bridging the gap between selfreported and claims-derived adherence measures for basal insulin among individuals with T2DM [abstract]. J Manag Care Spec Pharm. 2017;23(10-a Suppl):S34. Available at: https://www.jmcp.org/doi/pdf/10.18553/ jmcp.2017.23.10-a.s1.

27. Bureau of Labor Statistics. CPI-all urban consumers (current series). Retreived March 12, 2018. Available at: https://data.bls.gov/cgi-bin/ surveymost?bls. Accessed June 19, 2019.

28. Young BA, Lin E, Von Korff M, et al. Diabetes complications severity index and risk of mortality, hospitalization, and healthcare utilization. Am J Manag Care. 2008;14(1):15-23.

29.Quan H, Sundararajan V, Halfon P, et al. Coding algorithms for defining comorbidities in ICD-9-CM and ICD-10 administrative data. Med Care. 2005;43(11):1130-39.

30. Yang D, Dalton JE. A unified approach to measuring the effect between two groups using SAS. SAS Global Forum 2012. Available at: http://support.sas.com/ resources/papers/proceedings12/335-2012.pdf. Accessed May 30, 2019.

31. Austin PC. The performance of different propensity score methods for estimating marginal hazard ratios. Stat Med. 2013;32(16):2837-49.

32. Rosenbaum PR, Rubin DB. The central role of the propensity score in observational studies for causal effects. Biometrika. 1983;70(1):41-55.

33. National Center for Chronic Disease Prevention and Health Promotion National diabetes statistics report, 2017. Available at: https://www.cdc.gov/ diabetes/pdfs/data/statistics/national-diabetes-statistics-report.pdf. Accessed May 30, 2019.

34. D'Agostino RB Jr. Propensity score methods for bias reduction in the comparison of a treatment to a non-randomized control group. Stat Med. 1998;17(19):2265-81.

35. Giorgino F, Bonadonna RC, Gentile S, Vettor R, Pozzilli P. Treatment intensification in patients with inadequate glycemic control on basal insulin: rationale and clinical evidence for the use of short-acting and other glucagonlike peptide-1 receptor agonists. Diabetes Metab Res Rev. 2016;32(6):497-511.

36. Yacoub T. Impact of improving postprandial glycemic control with intensifying insulin therapy in type 2 diabetes. Postgrad Med. 2017;129(8):791-800.

37. Holman RR, Farmer AJ, Davies MJ, et al. Three-year efficacy of complex insulin regimens in type 2 diabetes. N Engl J Med. 2009;361(18):1736-47.

38. Asche CV, Bode B, Busk AK, Nair SR. The economic and clinical benefits of adequate insulin initiation and intensification in people with type 2 diabetes mellitus. Diabetes Obes Metab. 2012;14(1):47-57.

39. García-Pérez LE, Alvarez M, Dilla T, Gil-Guillen V, Orozco-Beltrán D. Adherence to therapies in patients with type 2 diabetes. Diabetes Ther. 2013;4(2):175-94

40. Peyrot M, Barnett AH, Meneghini LF, Schumm-Draeger PM. Factors associated with injection omission/non-adherence in the Global Attitudes of Patients and Physicians in Insulin Therapy study. Diabetes Obes Metab. 2012;14(12):1081-87.

41. Peyrot M, Rubin RR, Kruger DF, Travis LB. Correlates of insulin injection omission. Diabetes Care. 2010;33(2):450-52.

42. Boonin A, Balinski B, Sauter J, Martinez J, Abbott S. A retrospective chart review of two different insulin administration systems on glycemic control in older adults in long-term care. J Gerontol Nurs. 2017;43(1):10-16. 
Effect of V-Go Versus Multiple Daily Injections on Glycemic Control, Insulin Use, and Diabetes Medication Costs Among Individuals with Type 2 Diabetes Mellitus

\section{APPENDIX A Codes Used to Identify Medications}

Medications of Interest

V-Go Wearable Insulin Delivery device

Insulin preparations

\begin{tabular}{l|}
\hline Any insulin \\
\hline Rapid-acting (bolus) \\
\hline Aspart \\
\hline Lispro \\
\hline Glulisine \\
\hline Inhaled insulin \\
\hline Insulin regular \\
\hline Long-acting (basal) \\
\hline Glargine \\
\hline Detemir \\
\hline Degludec
\end{tabular}

Oral antidiabetic medication

Biguanides

DPP-4 inhibitors

Thiazolidinediones

Sulfonylureas

SGLT2 inhibitors

Noninsulin injectables

GLP-1 receptor agonists

Insulin pumps

Antihypertensive agents

Lipid-lowering agents

Cardiovascular medications

DPP-4 = dipeptidyl peptidase

NDC = National Drug Code; NPH = neutral protamine Hagedorn; SGLT2 = sodium-glucose co-transporter-2.

$27104003 x, 27104006 x, 2710400700 D 2 x x$

$2710400700 \mathrm{D} 2 \mathrm{x}$

$27104070 x, 27104080 x, 27104090 x$

2755x, 279965x, 279940x, 279930x, 279925x

$78 x, 279940 x$

$2720 x, 279978 x, 279970 x$

2717x, 279910x, 6125205000D220

NDC: 08508114002,08508112010

$33 \mathrm{x}, 34 \mathrm{x}, 36 \mathrm{x}, 37 \mathrm{x}, 409925021503 \mathrm{xx}$
GPI/NDC/HCPCS Codes

NDC: 08560940001, 08560940002,08560940003

\begin{tabular}{|l}
\hline $2710 x$ \\
\hline $27104002 x, 27104005 x, 27104004 x, 271040100029,27104010 x, 27104015 x, 27104030 x$ \\
\hline $27104002 x$ \\
\hline $27104005 x$ \\
\hline $27104004 x$ \\
\hline $271040100029 x$ \\
\hline $27104010 x, 27104015 x, 27104030 x$ \\
\hline $27104003 x, 27104006 x, 2710400700 D 2 x x$ \\
\hline $27104003 x$ \\
\hline $27104006 x$ \\
\hline $2710400700 D 2 x$ \\
\hline $27104070 x, 27104080 x, 27104090 x$ \\
\hline $2710401000 D 250,27104010002015,27103010002015$ \\
\hline $2725 x, 279925 x, 279950 x, 279960 x, 279970 x, 279980 x$ \\
\hline $2755 x, 279965 x, 279940 x, 279930 x, 279925 x$ \\
\hline $2760 x, 279980 x, 279978 x, 279940 x$ \\
\hline $2720 x, 279978 x, 279970 x$ \\
\hline $2770 x, 279960 x, 279965 x$ \\
\hline
\end{tabular}

$27104002 x, 27104005 x, 27104004 x, 271040100029,27104010 x, 27104015 x, 27104030 x$

$2725 x, 279925 x, 279950 x, 279960 x, 279970 x, 279980 x$

GPI. $97201030006200,97201030006400,97201030106300,97201030206300,97201030506300$

8560x, 3210x, 8310x, 8320x, 8330x, 8333x, 8337x, 3940x, 8520x, 8515x, 6410x, 6499x

peptide-1; GPI = Generic Product Identifier; HCPCS = Healthcare Common Procedure Coding System

APPENDIX B Baseline Demographic and Clinical Characteristics and Medication Use Among V-Go and MDI Users: Before and After Matching for Sensitivity Analysis

\begin{tabular}{|c|c|c|c|c|c|}
\hline \multirow[b]{2}{*}{ Baseline Characteristics } & \multicolumn{3}{|c|}{ Cohorts Before Matching } & \multicolumn{2}{|c|}{ Cohorts After Matchinga } \\
\hline & V-Go Cohort & MDI Cohort & $\mathrm{SMD}^{\mathrm{b}}$ & MDI Cohort & SMD \\
\hline Sample size & 585 & 16,022 & & 585 & \\
\hline \multicolumn{6}{|l|}{ Demographic characteristics } \\
\hline Female, n (\%) & $301(51.5)$ & $7,093(44.3)^{c}$ & 0.14 & $296(50.6)$ & 0.02 \\
\hline Age at index, in years, mean (SD), median & $56.7(9.8), 58.0$ & $57.5(11.4), 58.0$ & -0.07 & $57.0(10.92), 58.0$ & -0.03 \\
\hline HMO plan, n (\%) & $149(25.5)$ & $3,533(22.1)$ & 0.08 & $122(20.9)$ & 0.11 \\
\hline PPO plan, n (\%) & $347(59.3)$ & $11,057(69.0)$ & -0.20 & $423(72.3)$ & -0.28 \\
\hline CDHP plan, n (\%) & $89(15.2)$ & $1,431 \quad(8.9)$ & 0.19 & $40(6.8)$ & 0.27 \\
\hline Commercial insurance type, $\mathrm{n}(\%)$ & $523(89.4)$ & $13,731 \quad(85.7)^{\mathrm{c}}$ & 0.11 & $507(86.7)$ & 0.08 \\
\hline \multicolumn{6}{|l|}{ Region, n (\%) } \\
\hline Northeast & $83(14.2)$ & $2,337(14.6)$ & -0.01 & $93(15.9)$ & -0.05 \\
\hline Midwest/West & $243(41.5)$ & $8,172(51.0)^{\mathrm{c}}$ & -0.19 & $295(50.4)^{\mathrm{c}}$ & -0.18 \\
\hline South & $259(44.3)$ & $5,513(34.4)^{c}$ & 0.20 & $197(33.7)^{\mathrm{c}}$ & 0.22 \\
\hline \multicolumn{6}{|l|}{ Physician specialty, ${ }^{\mathrm{d}} \mathrm{n}(\%)$} \\
\hline Endocrinologist & $275(47.0)$ & $4,947(30.9)^{c}$ & 0.34 & $231(39.5)^{c}$ & 0.15 \\
\hline PCP & $128(21.9)$ & $7,497(46.8)^{c}$ & -0.54 & $234(40.0)^{c}$ & -0.40 \\
\hline
\end{tabular}

continnued on next page 
APPENDIX B Baseline Demographic and Clinical Characteristics and Medication Use Among V-Go and MDI Users: Before and After Matching for Sensitivity Analysis (continued)

\begin{tabular}{|c|c|c|c|c|c|}
\hline \multirow[b]{2}{*}{ Baseline Characteristics } & \multicolumn{3}{|c|}{ Cohorts Before Matching } & \multicolumn{2}{|c|}{ Cohorts After Matchinga } \\
\hline & V-Go Cohort & MDI Cohort & $\mathrm{SMD}^{\mathrm{b}}$ & MDI Cohort & SMD \\
\hline \multicolumn{6}{|l|}{ Physician specialty, ${ }^{\mathrm{d}} \mathrm{n}(\%)$} \\
\hline Other & $20(3.4)$ & $1,431 \quad(8.9)^{\mathrm{c}}$ & -0.23 & $52(8.9)^{\mathrm{c}}$ & -0.23 \\
\hline Missing/unknown & $162(27.7)$ & $2,147(13.4)^{\mathrm{c}}$ & 0.36 & $68(11.6)^{c}$ & 0.41 \\
\hline \multicolumn{6}{|l|}{ Clinical conditions of interest } \\
\hline QCI score, mean (SD), median & $2.3(1.60), 2.0$ & 2.4 (1.79), 2.0 & -0.04 & $2.3(1.63), 2.0$ & -0.01 \\
\hline aDCSI score, mean (SD), median & $1.5(1.86), 1.0$ & $1.6(1.91), 1.0$ & -0.05 & $1.5(1.76), 1.0$ & $<0.01$ \\
\hline \multicolumn{6}{|l|}{ Diabetic complications, n (\%) } \\
\hline Retinopathy & $116(19.8)$ & $2,843(17.7)$ & 0.05 & $108(18.5)$ & 0.03 \\
\hline Neuropathy & $193(33.0)$ & $4,097(25.6)^{\mathrm{c}}$ & 0.16 & $194(33.2)$ & $<0.01$ \\
\hline Nephropathy & $121(20.7)$ & $3,086(19.3)$ & 0.04 & $114(19.5)$ & 0.03 \\
\hline Cerebrovascular & $26(4.4)$ & $966(6.0)$ & -0.07 & $23(3.9)$ & 0.03 \\
\hline Cardiovascular & $129(22.1)$ & $4,483(28.0)^{c}$ & -0.14 & $129(22.1)$ & $<0.01$ \\
\hline Peripheral vascular disease & $59(10.1)$ & $1,917(12.0)$ & -0.06 & $74(12.6)$ & -0.08 \\
\hline \multicolumn{6}{|l|}{ Other comorbidities, $\mathrm{n}(\%)$} \\
\hline Hypertension & $453(77.4)$ & $11,907(74.3)$ & 0.07 & $448(76.6)$ & 0.02 \\
\hline Dyslipidemia & $487(83.2)$ & $11,315(70.6)^{c}$ & 0.30 & $489(83.6)$ & -0.01 \\
\hline Mental illness & $127(21.7)$ & $3,678(23.0)$ & -0.03 & $124(21.2)$ & 0.01 \\
\hline \multicolumn{6}{|l|}{ Insulin use } \\
\hline \multicolumn{6}{|l|}{ Previous insulin exposure, $\mathrm{n}(\%)$} \\
\hline Basal-bolus insulin & $375(62.4)$ & $7,636(47.7)^{c}$ & 0.34 & $374(63.9)$ & $<0.01$ \\
\hline Basal insulin only & $144(24.6)$ & $7,686(48.0)^{\mathrm{c}}$ & -0.50 & $146(25.0)$ & -0.01 \\
\hline Premixed insulin only & $66(11.3)$ & $700(4.4)^{c}$ & 0.26 & $65(11.1)$ & 0.01 \\
\hline \multicolumn{6}{|l|}{ Fills of insulin, mean (SD), median } \\
\hline Basal insulin & $2.6(1.88), 2.0$ & $3.99(2.11), 4.0^{c}$ & 0.70 & $2.6(1.78), 2.0^{\mathrm{c}}$ & -0.08 \\
\hline Bolus insulin & $1.7(1.65), 1.0$ & $1.4(1.98), 0.0^{\mathrm{c}}$ & 0.12 & $1.5(1.78), 1.0$ & 0.07 \\
\hline Premixed insulin & $0.4(1.18), 0.0$ & $0.27(1.10), 0.0^{c}$ & 0.11 & $0.4(1.22), 0.0$ & -0.03 \\
\hline \multicolumn{6}{|l|}{ Baseline other medications } \\
\hline \multicolumn{6}{|l|}{ Oral diabetes therapy, $\mathrm{n}(\%)$} \\
\hline Biguanides & $303(51.8)$ & $6,837(42.7)^{c}$ & 0.18 & $301(51.5)$ & 0.01 \\
\hline Sulfonylureas & $120(20.5)$ & $3,667(22.9)$ & -0.06 & $134(22.9)$ & -0.06 \\
\hline DPP-4i & $93(15.9)$ & $2,266(14.1)$ & 0.05 & $76(13.0)$ & 0.08 \\
\hline SGLT2i & $64(10.9)$ & $299(1.9)^{c}$ & 0.38 & $13(2.2)^{\mathrm{c}}$ & 0.36 \\
\hline TZD & $33(5.6)$ & $950(5.9)$ & -0.01 & $56(9.6)^{c}$ & -0.15 \\
\hline Any of the above & $388(66.3)$ & $8,878(55.4)^{c}$ & 0.23 & $387(66.2)$ & $<0.01$ \\
\hline \multicolumn{6}{|l|}{ Injectable noninsulin therapy, n (\%) } \\
\hline GLP-1 receptor agonists & $127(21.7)$ & $1,498(9.3)^{\mathrm{c}}$ & 0.35 & $126(21.5)$ & $<0.01$ \\
\hline \multicolumn{6}{|l|}{ Other medications, n (\%) } \\
\hline Antihypertensives & $470(80.3)$ & $13,092(81.7)$ & -0.03 & $491 \quad(83.9)$ & -0.09 \\
\hline Antihyperlipidemics & $435(74.4)$ & $11,158(69.6)^{c}$ & 0.11 & $446(76.2)$ & -0.04 \\
\hline CVD medications $^{e}$ & $415(70.9)$ & $10,817 \quad(67.5)$ & 0.07 & $420(71.8)$ & -0.02 \\
\hline
\end{tabular}

aThe V-Go cohort after matching is not shown in the table because the pre- and postmatch V-Go cohorts were identical.

bStandardized mean differences were estimated as the difference in means or proportions divided by the standard error.

cIndicates that statistical comparison of V-Go versus MDI had a P value $<0.05$ (using either t-tests or chi-square tests).

dTreating/prescribing physician specialty was captured based on specialty recorded on index claim (for V-Go or bolus insulin), or a T2DM office visit claim closest to index date within \pm 90 days. PCP includes family/general, internal medicine, and geriatrics.

${ }^{e} \mathrm{CVD}$ medications include thrombolytic, nitroglycerine, clot-preventing drugs, and antiplatelets.

aDCSI = adapted Diabetes Complications Severity Index; CDHP = consumer-driven health plan; $C V D=$ cardiovascular disease; DPP-4i=dipeptidyl-peptidase-4 inhibitors;

GLP-1 = glucagon-like peptide-1; HMO = health maintenance organization; $P C P=$ primary care provider; $P P O=$ preferred provider organization; $Q C I=Q$ uan-Charlson Comorbidity Index; $S D=$ standard deviation; SGLT2i = sodium-glucose transporter-2 inhibitors; SMD = standardized mean difference; TZD = thiazolidinediones . 